\title{
Typicality of recurrence for Lorentz gases
}

\author{
MARCO LENCI *
}

October 2004

\begin{abstract}
It is a safe conjecture that most (not necessarily periodic) two-dimensional Lorentz gases with finite horizon are recurrent. Here we formalize this conjecture by means of a stochastic ensemble of Lorentz gases, in which i.i.d. random scatterers are placed in each cell of a co-compact lattice in the plane.

We prove that the typical Lorentz gas, in the sense of Baire, is recurrent, and give results in the direction of showing that recurrence is an almost sure property (including a zero-one law that holds in every dimension). A few toy models illustrate the extent of these results.
\end{abstract}

Mathematics Subject Classification: 37D50, 37A40, 60K37.

\section{Introduction}

A Lorentz gas (LG) is the billiard system in the complement of a union of disjoint bounded, regular, convex sets of the plane. Namely, a dimensionless particle moves with constant unit velocity until it hits one of the sets (henceforth 'scatterers'), at which point it undergoes an instantaneous Fesnel reflection, i.e., the angle of reflection equals the angle of incidence.

This dynamical system generalizes on the one hand the so-called 'Sinai billiard', in which the particle is confined to a bounded domain, and on the other hand the periodic Lorentz gas, in which the scatterer configuration is invariant for the action of a co-compact lattice in $\mathbb{R}^{2}$.

The most important feature that comes with a LG being an extended system is that its physically relevant measure, the Liouville measure, is infinite. In this paper we are interested in the most fundamental ergodic property of an infinite-measure system: Poincaré recurrence. This property is far from trivial, in general [A]. As a matter of fact, it took the community considerable effort and more than a decade to prove recurrence for periodic LGs with finite horizon (i.e., such that the free path

${ }^{*}$ Department of Mathematical Sciences, Stevens Institute of Technology, Hoboken, NJ 07030, U.S.A. E-mail: mlenci@math.stevens.edu 
between two collisions is bounded from above). This was achieved by Schmidt [Sch] and Conze [Co] at the end of the 1990's. Recurrence has earned further importance lately, as it was proved [L2] that it is a sufficient condition for ergodicity. (See Section 2 for the definition of ergodicity in infinite measure and for the general geometric assumptions that are needed for this and all the forthcoming results.) It is well known that, for dispersing billiards such as ours, ergodicity implies much stronger chaotic properties - in our case, for suitable finite-measure Poincaré maps. (Good surveys of old and recent results in this celebrated field are found in $[\mathrm{KS}]$, $[\mathrm{SC}],[\mathrm{CY}],[\mathrm{CM}])$.

The motivation behind the present work is the idea that "most" finite-horizon Lorentz gases must be recurrent. After all, if the most orderly scatterer configurations, the periodic ones, give rise to diffusive and thus recurrent dynamical systems, one imagines that the same must happen for the "typical" configuration. This conviction is corroborated by the results of [L2]. For instance, a LG can be nonrecurrent only if it is totally transient, i.e., almost all trajectories escape to infinity. Also, a compactly supported perturbation of a recurrent LG is recurrent as well. As a matter of fact, no example has been constructed yet of a transient LG.

In order to formalize the intuition above into a precise conjecture, we present a very natural space of LGs, an ensemble, in the sense that it comes endowed with a probability measure. Given a lattice with compact fundamental domain, we partition the plane into copies of this domain (henceforth 'cells'). In each cell we place a random configuration of scatterers so that the configurations in two distinct cells are independent and identically distributed. We call this ensemble $\mathcal{L}$.

The conjecture then reads: almost all gases in $\mathcal{L}$ are recurrent. We are not able to prove the conjecture as of now, but can give a list of results that will hopefully put it within closer reach (and anyway make it all the more credible). For instance, the set $\mathcal{R}$ of recurrent LGs is topologically typical in $\mathcal{L}$, provided one metrizes the latter in a reasonable way (cf. Section 3). Moreover, $\mathcal{R}$ has either full or zero measure (Section 4). This last result is particularly valuable as it generalizes to all dimensions.

We also construct a finite-measure dynamical system whose dynamics comprises that of all orbits in all configurations of $\mathcal{L}$. The almost sure recurrence in $\mathcal{L}$ is equivalent to the cocycle recurrence of a certain function over this system, and we give a sufficient condition for that (Section 5). Verifying the condition on our model, however, seems rather complicated, so we study how this dynamical system behaves for a few simple models (Section 6).

From a technical viewpoint, the paper builds on the results of [L2]. The reader, however, need not know the details of the proofs, but just the statements, which are given for convenience in Section 2.

Acknowledgments. I wish to thank Lai-Sang Young, François Ledrappier and Charles Newman for very useful discussions. This work was partially supported by NSF Grant DMS-0405439. Previous travel funding from GNFM (Italy) is also acknowledged. 


\section{Definitions and preliminary results}

Let $\left\{\mathcal{O}_{\alpha}\right\}_{\alpha \in \mathcal{I}}$ be a family of pairwise disjoint, open, bounded, convex subsets of $\mathbb{R}^{2}$, with $C^{3}$ boundary; $\mathcal{I}$, the index set, is assumed countable.

With the term 'Lorentz gas' (LG) we will indicate both the family $\left\{\mathcal{O}_{\alpha}\right\}$ (also called 'scatterer configuration' or simply 'configuration') and the billiard system in $\mathbb{R}^{2} \backslash \bigcup_{\alpha \in \mathcal{I}} \mathcal{O}_{\alpha}$. The following definitions, assumptions and basic facts regarding the billiard dynamics are standard - and, at any rate, described in larger detail in [L2] - therefore we will lay them out rather concisely.

To each scatterer one associates the cylinder $\mathcal{M}_{\alpha}:=S_{L_{\alpha}}^{1} \times[0, \pi]$, where $S_{L_{\alpha}}^{1}$ is the circle of circumference $L_{\alpha}$, the latter being the length of $\partial \mathcal{O}_{\alpha}$. A pair $(r, \varphi) \in$ $\mathcal{M}_{\alpha}$ represents the element $(q, v)$ of the unit tangent bundle of $\mathbb{R}^{2} \backslash \bigcup_{\alpha} \mathcal{O}_{\alpha}$ thus determined: $q$ is the point of $\partial \mathcal{O}_{\alpha}$ parametrized by the arc-length coordinate $r$ (an origin $r=0$ is fixed once and for all on every $\mathcal{O}_{\alpha}$, and $r$ increases when moving counterclockwise along $\left.\partial \mathcal{O}_{\alpha}\right) ; v \in \mathrm{T}_{q} \mathbb{R}^{2}$ is the unit vector based in $q$ that forms a counterclockwise angle $\varphi$ with the tangent line to $\mathcal{O}_{\alpha}$ at $q$, and points outwardly w.r.t. $\mathcal{O}_{\alpha}$ (see Fig. 1). In the rest of the paper will also denote pairs $(r, \varphi)$ by $x$.

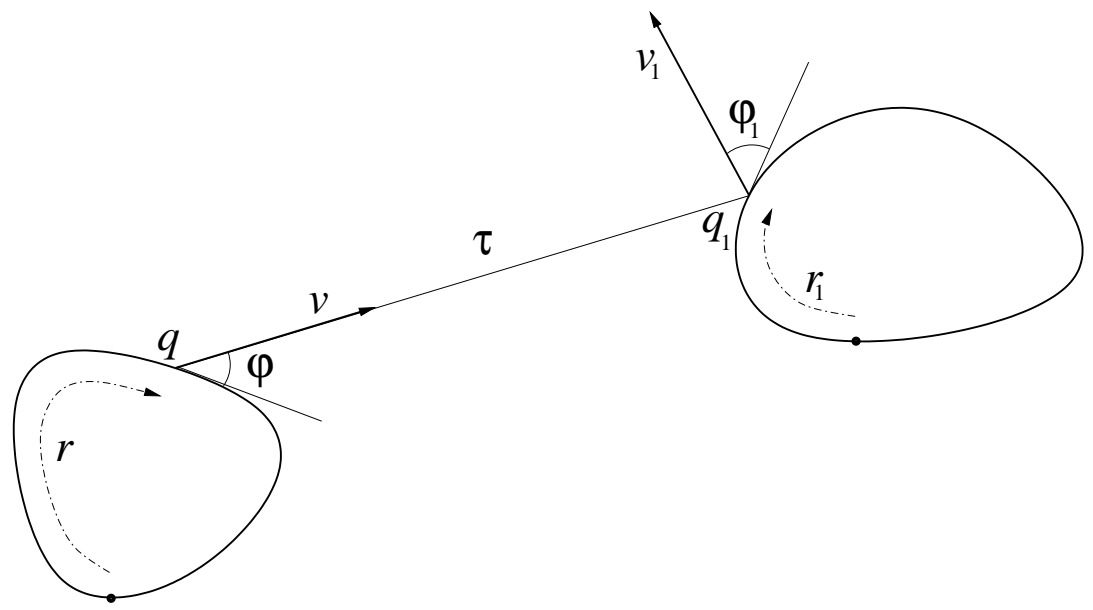

Figure 1: Basic definitions for the billiard map.

The phase space $\mathcal{M}:=\bigsqcup_{\alpha} \mathcal{M}$ is the disjoint union of all the $\mathcal{M}_{\alpha}$ (the disjoint union is a needed formality because points belonging to different $\mathcal{M}_{\alpha}$ may be denoted by the same pair $(r, \varphi))$. We introduce a map $T$ whose action is illustrated in Fig. 1: $T x=x_{1}$ if $x$ represents $(q, v), x_{1}$ represents $\left(q_{1}, v_{1}\right)$, and a material point in $q$ traveling with velocity $v$ has its first collision at $q_{1}$ with postcollisional velocity $v_{1}$. $T$, which is called the billiard map, preserves a measure $\mu$ on $\mathcal{M}$, defined by the density $d \mu(r, \varphi):=\sin \varphi d r d \varphi$. Clearly $\mu(\mathcal{M})=2 \sum_{\alpha} L_{\alpha}=\infty$, save for some pathological situations when the size of $\mathcal{O}_{\alpha}$ accumulates at zero. As a matter of fact, such situations will be explicitly excluded by the following assumptions, that we maintain throughout the paper. 
If $x$ represents $(q, v)$ with $q \in \mathcal{M}_{\alpha}$, let $k(x)$ denote the curvature of $\partial \mathcal{O}_{\alpha}$ in $q$, and $\tau(x)$ the free path of $x$, i.e., the distance between $q$ and $q_{1}$, the next collision point (see again Fig. 1). By hypothesis, there exist $k_{m}, k_{M}, \tau_{m}, \tau_{M}>0$, such that, $\forall x \in \mathcal{M}$,

$$
\begin{aligned}
& k_{m} \leq k(x) \leq k_{M} \\
& \tau_{m} \leq \tau(x) \leq \tau_{M} .
\end{aligned}
$$

The second inequality in (2.2) is the celebrated finite horizon condition. It is clear that (2.1) implies that the size of any $\mathcal{O}_{\alpha}$ is bounded above and below.

The following definitions may not be obvious for dynamical systems of infinite measure:

Definition 2.1 The measure-preserving dynamical system $(\mathcal{M}, T, \mu)$ is called (Poincaré) recurrent if, for every measurable $A \subseteq \mathcal{M}$, the orbit of $\mu$-almost every $x \in A$ returns to $A$ at least once (and thus infinitely many times, due to the invariance of $\mu$ ).

Definition 2.2 The measure-preserving dynamical system $(\mathcal{M}, T, \mu)$ is called er godic if every $A \subseteq \mathcal{M}$ measurable and invariant $\bmod \mu$ (i.e., $\mu\left(T^{-1} A \Delta A\right)=0$ ), has either zero measure or full measure (i.e., $\mu(\mathcal{M} \backslash A)=0$ ).

Dispersing billiards like the system at hand are prototypical examples of hyperbolic systems with singularities. The presence of the singularities represents a conspicuous hurdle in proving the hyperbolic and ergodic properties. This is even more so in infinite measure and, as is the case here, when the singularities themselves have an infinite extension (in the sense of their length as smooth curves in $\mathcal{M})$.

The following three results are the technical backbone of [L2]:

Theorem 2.3 The Lorentz gas introduced above has a hyperbolic structure, meaning that for $\mu$-a.e. $x \in \mathcal{M}$ there are local stable and unstable manifolds (LSUMs) at $x$, denoted $W^{s(u)}(x)$. These two measurable foliations (when endowed with a Lebesgue-equivalent transversal measure) are absolutely continuous w.r.t. $\mu$.

For a precise definition of LSUM in this context, see [L2]. Here we are primarily interested in their core property: if $y \in W^{s(u)}(x)$ then $d_{\mathcal{M}}\left(T^{n} x, T^{n} y\right) \rightarrow 0$ as $n \rightarrow$ $+\infty(-\infty) ; d_{\mathcal{M}}$ is the Riemannian distance in $\mathcal{M}$ (by definition $d_{\mathcal{M}}(x, y)=\infty$ if $x$ and $y$ belong to different cylinders $\left.\mathcal{M}_{\alpha}, \mathcal{M}_{\beta}\right)$. For our systems one can see that the rate of vanishing is exponential.

Theorem 2.4 Given $\alpha \in \mathcal{I}$, almost every two points $x, y \in \mathcal{M}_{a}$ are connected by a polyline of alternating LSUMs, in the sense that there is a finite collection of LSUMs $W^{s}\left(x_{1}\right), W^{u}\left(x_{2}\right), W^{s}\left(x_{3}\right), \cdots, W^{u}\left(x_{m}\right)$, with $x_{1}:=x$ and $x_{m}:=y$, such that each LSUM intersects the next transversally. 
Theorem $2.5(\mathcal{M}, T, \mu)$ is ergodic if and only if it is recurrent.

For this last theorem, only the sufficient condition was given in [L2], but the necessary condition is obvious, anyway: if there is a positive-measure wandering set, one can split it in two non-trivial parts, which must necessarily belong to two different ergodic components.

The following statement was used (and justified) in [L2], but never explicitly emphasized.

Proposition 2.6 A LG as introduced above is either recurrent, i.e., totally conservative, or totally dissipative.

Proof. The dissipative part $D$ of $(\mathcal{M}, T, \mu)$ is defined as the maximal countable union of wandering sets of $\mathcal{M}$, modulo $\mu[\mathrm{A}]$. If $\mu(D)>0$, we claim that $D$ contains whole LSUMs; that is, $W^{s}(D):=\left\{y \in W^{s}(x) \mid x \in D\right\}$ and the analog $W^{u}(D)$ are equal to $D$, modulo $\mu$. We prove that first statement, the second being obviously equivalent.

Take a positive-measure wandering set $A$. Without loss of generality, $A \subseteq \mathcal{M}_{\alpha}$ for some $\alpha \in \mathcal{I}$. Apart from a null-measure set, $A$ is the disjoint union of

$$
A_{n}:=\left\{x \in A \mid n=\max \left\{k \geq 0 \mid T^{k} x \in \mathcal{M}_{\alpha}\right\}\right\},
$$

with $n \geq 0$ (it is easy to see that almost no points of $A$ can return to $\mathcal{M}_{\alpha}$ infinitely many times). Pick $n$ for which $\mu\left(A_{n}\right)>0$. $W^{s}\left(T^{n} A_{n}\right)$ is a wandering set, because points in the same LSM have the same forward itinerary w.r.t. the partition $\left\{\mathcal{M}_{\beta}\right\}$ (that is, they hit the same scatterers in the future); in particular, if $y \in W^{s}(x)$ with $x \in T^{n} A_{n}$, then $T^{k} y \notin \mathcal{M}_{\alpha}$, for all $k>0$.

As is known, the local stable foliation can be chosen invariant, that is, $T W^{s}(x) \subseteq$ $W^{s}(T x)$ - this is in fact a standard assumption. Therefore $T^{n} W^{s}\left(A_{n}\right) \subseteq W^{s}\left(T^{n} A_{n}\right)$. Together with the above conclusions, this implies that $T^{n} W^{s}\left(A_{n}\right)$, and thus $W^{s}\left(A_{n}\right)$, is wandering. Repeating the argument for all $n$ such that $\mu\left(A_{n}\right)>0$ proves that $W^{s}(A)$ is wandering, yielding our initial claim.

By Theorem 2.4, then, any $\mathcal{M}_{\alpha}$ is either wholly contained in $D$ or in its complement. If $D \neq \mathcal{M}$, there must be two nearest neighbors $\mathcal{O}_{\alpha}$ and $\mathcal{O}_{\beta}$ such that $\mathcal{M}_{\alpha} \subseteq D$ and $\mathcal{M}_{\beta} \cap D=\emptyset$. But this is absurd as $D$ is $T$-invariant and there exists $B \subset \mathcal{M}_{\alpha}$, with $\mu(B)>0$, such that $T B \subset \mathcal{M}_{\beta}$.

Q.E.D.

In this paper we are interested in recurrent LGs, so let us start to give examples thereof. Recall the definition of periodic LG from the Introduction.

Theorem 2.7 [Sch, Co] A periodic LG with finite horizon and strictly convex scatterers is recurrent.

Definition 2.8 The $L G\left\{\mathcal{O}_{\alpha}\right\}_{\alpha \in \mathcal{I}}$ is called a finite modification of $\left\{\mathcal{O}_{\alpha}\right\}_{\alpha \in \mathcal{I}_{0}}$ if $\mathcal{I}=\left(\mathcal{I}_{0} \backslash \mathcal{I}_{1}\right) \cup \mathcal{I}_{2}$, where: 
(a) $\mathcal{I}_{1}$ is a finite subset of $\mathcal{I}_{0}$.

(b) $\mathcal{I}_{2}$ is the index set of a finite $L G$ such that $d_{\mathbb{R}^{2}}\left(\mathcal{O}_{\alpha}, \mathcal{O}_{\beta}\right)>0$ for any $\alpha \in \mathcal{I}_{2}$, $\beta \in \mathcal{I}_{0} \backslash \mathcal{I}_{1}\left(d_{\mathbb{R}^{2}}\right.$ is the distance in the plane).

Proposition 2.9 A LG is recurrent if and only if any of its finite modifications are recurrent.

Proof. The proof of the necessary condition is identical to that of Proposition 5.3 of [L2]. The sufficient condition follows automatically since a LG is a finite modification of any of its finite modifications.

Q.E.D.

\section{Model and topological typicality}

As explained in the Introduction, it is reasonable to conjecture that most LGs are recurrent. We need a satisfyingly general class of gases for which 'most' can be properly defined. Our choice is explained hereafter.

Consider a co-compact lattice $\Gamma \subset \mathbb{R}^{2}$, with $\left\{C_{\gamma}\right\}_{\gamma \in \Gamma}$ its corresponding partition of the plane, that is, $\mathbb{R}^{2}=\bigcup_{\gamma \in \Gamma} C_{\gamma}$, with $C_{\gamma}=C_{0}+\gamma$, and $C_{\gamma} \cap C_{\eta}=\emptyset$ for $\gamma \neq \eta$. In each cell $C_{\gamma}$ we put a random configuration of scatterers parametrized by $\ell_{\gamma}$, where $\left\{\ell_{\gamma}\right\}$ are independent identically distributed random variables from the same probability space $(\Omega, \pi)$. (In the remainder, a generic element of $\Omega$ with be denoted by $\omega$.) We assume that (2.1)-(2.2) are satisfied for every realization of this random field.

Examples are illustrated by Fig. 2 and its caption.

The class of LGs we will concern ourselves with is $(\mathcal{L}, \Pi):=(\Omega, \pi)^{\Gamma}$, where the superscript denotes the product of $\Gamma$ copies of $(\Omega, \pi)$. From now on a Lorentz gas will be an element $\ell=\left\{\ell_{\gamma}\right\} \in \mathcal{L}$.

In many cases, just as in the examples of Fig. $2, \Omega$ is also a metric space. We ask that the metric verifies the following natural property.

Definition 3.1 A distance function $d_{\Omega}$ on pairs of $\Omega$ is called compatible with the dynamics if:

(a) $\left(\Omega, d_{\Omega}\right)$ is a compact metric space.

(b) Every scatterer $\mathcal{O}^{(i)}(\omega)(i=1, \ldots, N)$ represented by $\omega \in \Omega$ depends in a $C^{3}$ fashion on $\omega$. In other words, if $C \subset \mathbb{R}^{2}$ is a cell and $\xi_{\omega}^{(i)}: S^{1} \longrightarrow C$ is the arclength parametrization of $\partial \mathcal{O}^{(i)}(\omega)$, renormalized to 1 , then $\left\|\xi_{\omega}^{(i)}-\xi_{\omega^{\prime}}^{(i)}\right\|_{C^{3}\left(S^{1}\right)} \rightarrow$ 0 , when $d_{\Omega}\left(\omega, \omega^{\prime}\right) \rightarrow 0$. 


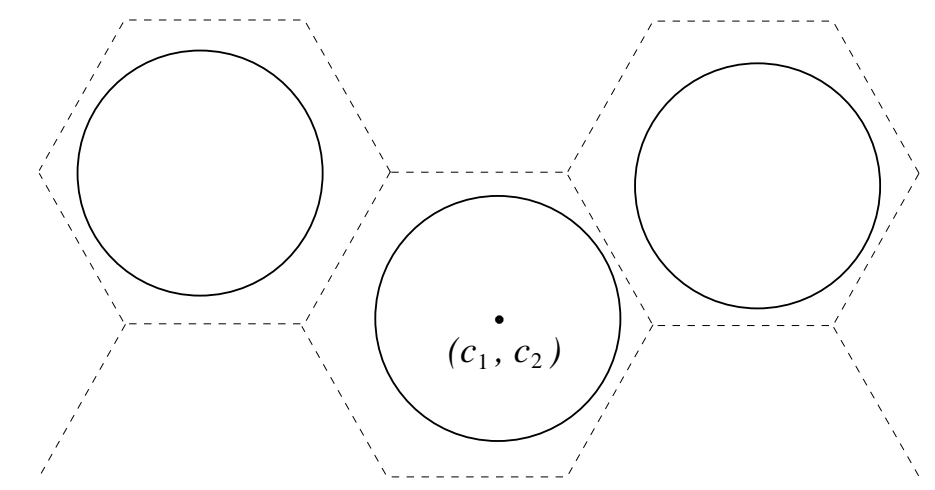

(a)

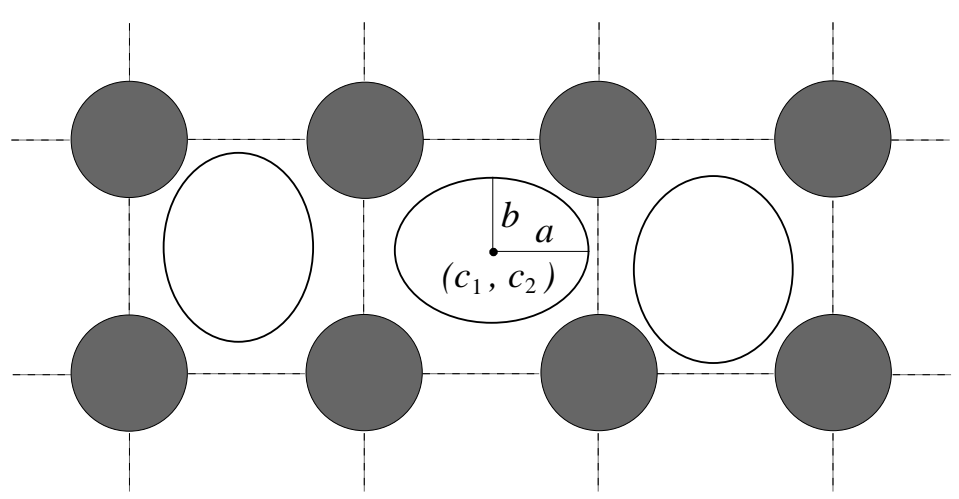

(b)

Figure 2: Two examples of random LGs. In (a), $\Gamma=$ Hex; in each cell the scatterer is a disc of radius $R$ and random center $\left(c_{1}, c_{2}\right)=: \omega \in \Omega:=$ $B(0, r)$, with $r$ sufficiently small; $\pi$ is the normalized Lebesgue measure on $\Omega$. In (b), $\Gamma=\mathbb{Z}^{2}$; the scatterer is an ellipse of random center $\left(c_{1}, c_{2}\right)$ and random semiaxes $a, b$, with $\omega:=\left(c_{1}, c_{2}, a, b\right) \in B(0, r) \times I_{1} \times I_{2}=: \Omega$ $\left(I_{1}, I_{2}\right.$ are intervals); $\pi$ is the normalized Lebesgue measure on $\Omega$; the gray, non-random scatterers are needed to comply with the finite-horizon condition.

Obviously, if $\Omega$ is finite, then $d_{\Omega}$ is compatible with the dynamics.

The above definition induces a distance on $\mathcal{L}$ which makes it a complete metric space. Namely, for $\ell=\left\{\ell_{\gamma}\right\}$ and $\ell^{\prime}=\left\{\ell_{\gamma}^{\prime}\right\}$,

$$
d_{\mathcal{L}}\left(\ell, \ell^{\prime}\right)=\sum_{\gamma \in \Gamma} 2^{-|\gamma|} d_{\Omega}\left(\ell_{\gamma}, \ell_{\gamma}^{\prime}\right)
$$

In this setup, recurrence is a typical property, in the sense of Baire:

Theorem 3.2 If $d_{\Omega}$ is compatible with the dynamics, then

$$
\mathcal{R}:=\{\ell \in \mathcal{L} \mid \ell \text { is a recurrent } \mathrm{LG}\}
$$

contains $a G_{\delta}$-set. 
Proof. We simplify the proof somewhat if we consider the cylinder

$$
\mathcal{L}_{0}:=\left\{\ell=\left\{\ell_{\gamma}\right\} \mid \ell_{0}=\omega_{0}\right\},
$$

where $\omega_{0}$ is a fixed element of $\Omega$. In view of Proposition 2.9, Theorem 3.2 is equivalent to showing that $\mathcal{R}_{0}:=\mathcal{R} \cap \mathcal{L}_{0}$ is a residual set of $\mathcal{L}_{0}$ in the appropriate topology.

First of all, let us construct a countable subset of $\mathcal{R}_{0}$ that is dense in $\mathcal{L}_{0}$. Take a dense sequence $\left\{\omega_{j}\right\}_{j \in \mathbb{N}}$ in $\Omega$ (if $\Omega$ is finite, one can use $\Omega$ instead of $\left\{\omega_{j}\right\}$ ). Let $\Lambda$ denote a finite subset of $\Gamma \backslash\{0\}$, and $\left\{j_{\eta}\right\}_{\eta \in \Lambda} \in \mathbb{N}^{\Lambda}$ an $m$-tuple of natural numbers indexed by the elements of $\Lambda$ (here $m=\# \Lambda)$. To each pair $\left(\Lambda,\left\{j_{\eta}\right\}\right)=: n$ is associated the configuration $\ell^{(n)}$, defined by

$$
\ell_{\gamma}^{(n)}=\left\{\begin{array}{cl}
\omega_{j_{\gamma}}, & \text { if } \gamma \in \Lambda ; \\
\omega_{0}, & \text { if } \gamma \notin \Lambda .
\end{array}\right.
$$

Since the set of such pairs $n$ is countable, let us pretend that $n \in \mathbb{N}$. By looking at definition (3.1), it is rather clear that $\left\{\ell^{(n)}\right\}_{n \in \mathbb{N}}$ is dense in $\mathcal{L}$. Furthermore, each $\ell_{n}$ is a finite modification of a periodic LG; hence $\ell^{(n)} \in \mathcal{R}_{0}$.

Now, for any $\ell$, let us consider a specific scatterer $\mathcal{O}_{0}$ in the cell $C_{0}$; for instance, $\mathcal{O}_{0}=\mathcal{O}^{(1)}\left(\omega_{0}\right)$ (which, in the notation of Definition 3.1(b), means the "first" scatterer of $C_{0}$ ). The crucial point is that $\mathcal{O}_{0}$ is exactly the same for every $\ell \in \mathcal{L}_{0}$, because the configuration in $C_{0}$ is fixed. We naturally call $\mathcal{M}_{0}$ the cylinder in phase space corresponding to $\mathcal{O}_{0}$. Also, set $\mu_{0}(\cdot):=\mu(\cdot) / \mu\left(\mathcal{M}_{0}\right)$.

If $\mathcal{B}(\ell, \rho) \subset \mathcal{L}_{0}$ denotes the ball of center $\ell$ and radius $\rho>0$, w.r.t. $d_{\mathcal{L}}$, we contend that for every $n, m \in \mathbb{N}$ there exists $\varepsilon_{m}^{(n)}>0$ such that, for all $\ell \in \mathcal{B}\left(\ell^{(n)}, \varepsilon_{m}^{(n)}\right)$ the set

$$
A(\ell):=\left\{x \in \mathcal{M}_{0} \mid \exists k>0 \text { such that } T_{\ell}^{k} x \in \mathcal{M}_{0}\right\}
$$

has measure

$$
\mu_{0}(A(\ell)) \geq\left(1-\frac{1}{m}\right)
$$

(In (3.4), $T_{\ell}$ represents the billiard map for the LG $\ell$.)

It is not too hard to verify this claim, once we have unraveled its rather intricated formulation. In fact, $\ell^{(n)}$ is recurrent and thus $A\left(\ell^{(n)}\right)$ has full measure in $\mathcal{M}_{0}$. Take then $x \in A\left(\ell^{(n)}\right)$, with $k>0$ its first return time to $\mathcal{M}_{0}$. The trajectory of $x$ up to $T_{\ell^{(n)}}^{k} x$ is non-singular in the sense that its polyline representation on $\mathbb{R}^{2} \backslash \bigcup_{\alpha} \mathcal{O}_{\alpha}$ is tangent to no $\mathcal{O}_{\alpha}$ (by convention, singular trajectories, a null-measure set, are ignored, at least after they hit the tangency). Therefore, if one slightly modifies the shape and location of the scatterers of $\ell^{(n)}$ (thus turning it into some $\ell$ with $\left.d_{\mathcal{L}}\left(\ell, \ell^{(n)}\right)<\varepsilon\right)$, then the sequence of scatterers hit by $\left\{T_{\ell}^{j} x\right\}_{j=0}^{k}$ is the same as for $\left\{T_{\ell^{(n)}}^{j} x\right\}_{j=0}^{k}$. In particular $T_{\ell}^{k} x \in \mathcal{M}_{0}$. (For the cogniscenti: $x \in \mathcal{M}_{0}$ will have the same forward itinerary up to time $k$, w.r.t. the partition $\left\{\mathcal{M}_{\alpha}\right\}$, if the perturbation of the LG, which induces a perturbation on the singularity set $\mathcal{S}$, leaves $x$ in the same connected component of $\mathcal{M}_{0} \backslash \mathcal{S}_{k}$, where $\mathcal{S}_{k}:=\mathcal{S} \cap T^{-1} \mathcal{S} \cap \cdots \cap T^{-k+1}$. Sufficiently small perturbations of $\ell^{(n)}$ will obviously do this.) 
The above reasoning shows that, for all $x \in A\left(\ell^{(n)}\right)$, there exists $\varepsilon=\varepsilon(x)>0$ such that, $\forall \ell \in \mathcal{B}\left(\ell^{(n)}, \varepsilon\right), x \in A(\ell)$, too. Whence the claim.

Finally, the set

$$
\mathcal{G}:=\bigcap_{m \in \mathbb{N}} \bigcup_{n \in \mathbb{N}} \mathcal{B}\left(\ell^{(n)}, \varepsilon_{m}^{(n)}\right)
$$

is $G_{\delta}$ by construction. From $(3.5), \mu_{0}(A(\ell))=1$, for all $\ell \in \mathcal{G}$. This proves Theorem 3.2 because, for such $\ell$, it follows that $\mathcal{M}_{0}$ belongs in the conservative part of $\left(\mathcal{M}_{\ell}, T_{\ell}, \mu\right)$ (with the obvious meaning for $\mathcal{M}_{\ell}$ ). Therefore, by Proposition 2.6, $\ell \in \mathcal{R}_{0}$.

Q.E.D.

Remark 3.3 It is evident that the lattice structure of $\mathcal{L}$ played essentially no role in the proof of Theorem 3.2. Using the same method, one can prove the same result for any complete metric space $\mathcal{X}$ of LGs such that:

- $\mathcal{X}$ has a dense set of recurrent gases (e.g., finite modifications of periodic LGs).

- The distance is compatible with the dynamics, that is, two configurations $\ell, \ell^{\prime} \in \mathcal{X}$ are close if and only if there is a bijective correspondence between their scatterers such that corresponding scatterers have $C^{3}$-uniformly close boundaries.

\section{Measure-theoretic typicality}

We are aiming for a stronger notion of typicality for the recurrence property, as the space $\mathcal{L}$ was constructed with a built-in probability measure $\Pi$.

Conjecture $4.1 \Pi(\mathcal{R})=1$.

This seems very credible, especially in light of Theorem 2.7: if a periodic configuration produces a recurrent dynamics, then a typical random configuration will randomize the motion of the particle even more, making it possibly even more similar to a random walk.

Unfortunately, Conjecture 4.1 will remain such throughout the paper. The following result, however, seems to indicate that we are on the right track.

Theorem 4.2 If $\mathcal{A}$ is the $\sigma$-algebra induced on $\mathcal{L}$ by its construction (i.e., $\mathcal{A}=\mathcal{C}^{\otimes \Gamma}$, where $\mathcal{C}$ is the $\sigma$-algebra defined on $\Omega$ ), then $\mathcal{R} \in \overline{\mathcal{A}}^{\Pi}$ and $\Pi(\mathcal{R}) \in\{0,1\}$.

Proof. The second assertion is rather trivial once we establish the first. In fact, consider this natural action of $\Gamma$ on $\mathcal{L}$ : for $\eta \in \Gamma$,

$$
\sigma_{\eta}(\ell)=: \ell^{\prime}=\left\{\ell_{\gamma}^{\prime}\right\}_{\gamma \in \Gamma}, \quad \text { with } \quad \ell_{\gamma}^{\prime}:=\ell_{\gamma+\eta} .
$$


Obviously, $\sigma$ preserves the measure $\Pi$. Furthermore, $\sigma_{\eta}(\mathcal{R})=\mathcal{R}$ for all $\eta \in \Gamma$, since recurrence is a translation invariant property. On the other hand, $\left(\mathcal{L},\left\{\sigma_{\eta}\right\}_{\eta \in \Gamma}, \Pi\right)$ is ergodic (it is by definition a generalized Bernoulli shift in two dimensions). These two facts imply that $\Pi(\mathcal{R}) \in\{0,1\}$.

For the first statement we use the same trickery as in the proof of Theorem 3.2. From Proposition 2.9 we know that $\mathcal{R}$ is invariant w.r.t. changes in the $0^{\text {th }}$ component (i.e., $\ell \in \mathcal{R} \Longleftrightarrow \ell^{\prime} \in \mathcal{R}$, for all $\ell^{\prime}$ such that $\ell_{\gamma}^{\prime}=\ell_{\gamma}$, whenever $\gamma \neq 0$ ). More in detail, it is a "cylinder" whose sections are the $\mathcal{R}_{0}$ introduced in the proof of Theorem 3.2 (one for each $\omega_{0}$ ). If we call $\mathcal{A}_{0}$ and $\Pi_{0}$, respectively, the factor $\sigma$-algebra and the factor measure induced by $\mathcal{A}$ and $\Pi$ on the cylinder $\mathcal{L}_{0}$ (notice that $\left.\left(\mathcal{L}_{0}, \mathcal{A}_{0}, \Pi_{0}\right) \simeq(\Omega, \mathcal{C}, \pi)^{\Gamma \backslash\{0\}}\right)$, then

$$
\mathcal{R}_{0} \in{\overline{\mathcal{A}_{0}}}^{\Pi_{0}} \quad \Longrightarrow \quad \mathcal{R} \in \overline{\mathcal{A}}^{\Pi} .
$$

As for proving the above l.h.s., we recall the definition of $\mathcal{M}_{0}$ from the proof of Theorem 3.2, and set

$$
A:=\left\{(x, \ell) \in \mathcal{M}_{0} \times \mathcal{L}_{0} \mid \limsup _{k \rightarrow+\infty}\left(\chi_{\mathcal{M}_{0}} \circ T_{\ell}^{k}\right)(x)=1\right\} .
$$

$A$ is measurable because $T_{\ell} x$ is clearly a measurable function of $(x, \ell)$ (indeed, due to the finite-horizon condition, it does not depend on the scatterers of $\ell$ that are at a certain distance from $\mathcal{O}_{0}$; so it is even measurable w.r.t. a certain subalgebra of sets depending only on a finite number of lattice sites).

Now, Proposition 2.6 implies that, for any given $\ell$, either a full-measure or a zeromeasure set of points in $\mathcal{M}_{0}$ come back to $\mathcal{M}_{0}$ infinitely many times, depending on $\ell$ being recurrent or not. This amounts to saying that, almost surely, $A$ contains whole "horizontal" fibers of $\mathcal{M}_{0} \times \mathcal{L}_{0}$, that is, $A=\mathcal{M}_{0} \times \mathcal{R}_{0} \bmod \mu \times \Pi$. By Lemma A.1 of the Appendix, $\mathcal{R}_{0} \in \overline{\mathcal{A}}_{0}{ }^{\Pi}$.

Q.E.D.

Remark 4.3 Theorem 4.2 is much more general than was presented here, and applies easily to the $d$-dimensional case. In fact, the only non-trivial ingredient in the proof is Proposition 2.6, which is in turn a consequence of Theorem 2.4 (that is just a weak formulation of the local ergodicity theorem). Therefore, if (2.1) is substituted by

$$
k_{m} \leq \mathbf{k}(q) \leq k_{M},
$$

where $\mathbf{k}(q)$ is the second fundamental form of $\partial \mathcal{O}_{\alpha}$ at $q$ (the inequalities here are meant in the sense of the quadratic forms), then Theorem 4.2 holds for the class $\mathcal{L}=\mathcal{L}(d, \Gamma, \Omega, \pi)$ of $d$-dimensional LGs with i.i.d. random scatterers in every cell of $\Gamma$, selected from the probability space $(\Omega, \pi)$, whenever the geometry of the scatterers makes the local ergodicity theorem hold. This includes at least all semi-dispersing scatterers given by algebraic equations [BCST]. Moreover, one can apply this zeroone law to many situations in which the dimension of $\Gamma$ is strictly less than the dimension of the Euclidean space (e.g., a 3D billiard in an infinite parallelepiped acted upon by $\mathbb{Z}$, and so on...). 


\section{A finite-measure dynamical system}

From a technical point of view, the difficulties associated with our system arise by and large from the fact that the given invariant measure has infinite mass. But the lattice structure of $\mathcal{L}$ suggests the construction of a finite-measure dynamical system that embodies all LGs in $\mathcal{L}$.

Consider the cell $C_{0}$ associated to the origin of $\Gamma$ : we think of it as our fundamental domain. Call $\partial_{*} C_{0}$ the part of $\partial C_{0}$ that does not intersect any non-random scatterer $\left(\partial C_{0}\right.$ can never intersect a random scatterer, anyway, lest (2.2) be violated; as a matter of fact, the random scatterers must keep at least $\tau_{m} / 2$ units away from $\partial C_{0}$ ). In example (a) of Fig. $2, \partial_{*} C_{0}=\partial C_{0}$, whereas in example (b), $\partial_{*} C_{0}$ is the union of four disjoint segments of equal length. Define

$$
\mathcal{N}:=\left\{(q, v) \in \mathrm{TR}^{2}\left|q \in \partial_{*} C_{0},\right| v \mid=1 \text {, and } v \text { points inwardly w.r.t. } C_{0}\right\} .
$$

To maintain consistency with the notation of Section 2, we identify $\partial_{*} C_{0}$ with a subset $J$ of $\mathbb{R}$, in which an arc-length coordinate $r$ uniquely determines a point $q \in \partial_{*} C_{0}$. Then, if $\varphi$ parametrizes the direction of $v$ in the usual way (like in Fig. 1), then $\mathcal{N}$ can be identified with $J \times[0, \pi]$.

Remark 5.1 This identification is always flawed at a finite number of points in $\partial_{*} C_{0}$. For instance, in Fig. 2(a), at the six vertices of $\partial C_{0}$; in Fig. 2(b), at the eight boundary points of $\partial_{*} C_{0}$. There are two ways to do away with this problem. The first way is tantamount to ignoring it: one can exclude these points from $\partial_{*} C_{0}$ (in which case, $\partial_{*} C_{0}$ will always be a disjoint union of open intervals). This exclusion is acceptable since it affects only a null-measure subset of $\partial_{*} C_{0}$, w.r.t. the relevant measure that we introduce below. The second way consists in identifying, on a case-by-case basis, different pairs $(r, \varphi)$ and $\left(r_{1}, \varphi_{1}\right)$, corresponding either to the same line element, or to the pre- and post-collisional line elements for the same collision. For instance, in example (b), if $r_{0}$ is the left endpoint of an interval of $\partial_{*} C_{0},\left(r_{0}, \varphi\right) \simeq\left(r_{0}, \pi-\varphi\right)$.

Let us call $\mu_{1}$ the standard billiard-invariant measure for the cross-section $\mathcal{N}$, normalized to 1 (in $(r, \varphi)$ coordinates, $d \mu_{1}(r, \varphi)=\left[2 \operatorname{length}\left(\partial_{*} C_{0}\right)\right]^{-1} \sin \varphi d r d \varphi$ ). If $\omega \in \Omega$ determines the configuration of scatterers in $C_{0}$, we can define a map $R_{\omega}: \mathcal{N} \longrightarrow \mathcal{N}$ as follows. Trace the (forward) trajectory of $x:=(q, v) \in \mathcal{N}$ until it crosses $\partial C_{0}$ for the first time - see Fig. 3. This occurs at the point $q_{1}$ and with velocity $v_{1}$. Say that $C_{\gamma}$ is the cell that the particle enters upon leaving $C_{0}$. Define then

$$
\begin{aligned}
R_{\omega} x=R_{\omega}(q, v) & :=\left(q_{1}-\gamma, v_{1}\right) \in \mathcal{N} ; \\
e(x, \omega) & :=\gamma \in G .
\end{aligned}
$$

Here $G \subset \Gamma$ is the set of primitive directions of $\Gamma$, each corresponding to a neighboring cell of $C_{0}$. We name $e$ the 'exit function'. Finally, $R_{\omega}$ preserves $\mu_{1}$. (To give 
but a brief explanation, $\partial_{*} C_{0}$ is a transparent wall for the billiard flow. Poincaré maps for transparent walls are virtually the same as those for reflecting walls - they are actually a commonly used trick in billiard dynamics, cf. [L1].)

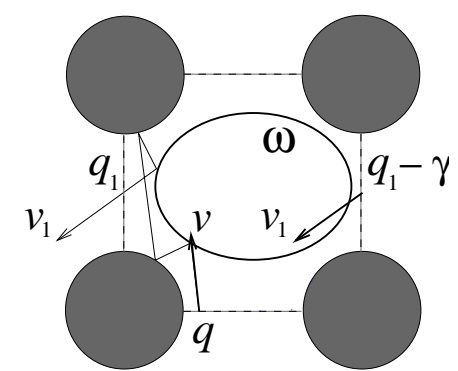

Figure 3: The definition of $R_{\omega}$. In this case $R_{\omega}(q, v)=\left(q_{1}-\gamma, v_{1}\right)$, with $\gamma=(-1,0) \in \mathbb{Z}^{2}$.

The dynamical system that we want to introduce in this section is the triple $(\Sigma, F, \nu)$, where:

- $\Sigma:=\mathcal{N} \times \mathcal{L}$.

- $F(x, \ell):=\left(R_{\ell_{0}} x, \sigma_{e\left(x, \ell_{0}\right)}(\ell)\right)$, defining a map $\Sigma \longrightarrow \Sigma$. Here $\sigma$ is the $\Gamma$-action on $\mathcal{L}$ defined by (4.1) and $\ell_{0}$ is, as usual, the $0^{\text {th }}$ component of $\ell$.

- $\nu:=\mu_{1} \times \Pi$. Since $\mu_{1}$ is $R_{\omega}$-invariant for every $\omega \in \Omega$, and $\Pi$ is $\sigma$-invariant, then $\nu$ is $F$-invariant.

The idea behind this definition is that, instead of following a given orbit form a cell to another, every time we shift the LG in the direction opposite to the orbit displacement, so that the point always lands in $C_{0}$. Clearly, $F: \Sigma \longrightarrow \Sigma$ encompasses the dynamics of all points on all LGs of $\mathcal{L}$. It is equally as clear that we are in the case in which a.e. $\ell \in \mathcal{L}$ is recurrent if and only if the function $e$ verifies the following:

Definition 5.2 Let $(\Sigma, F, \nu)$ be a measure-preserving dynamical system with $\nu(\Sigma)=$ 1. If $e: \Sigma \longrightarrow \Gamma \subseteq \mathbb{R}^{d}$, define the cocycle

$$
S_{n}(z):=\sum_{k=0}^{n-1}\left(e \circ F^{k}\right)(z) .
$$

The function e (or the cocycle $S_{n}$ ) is called recurrent if, for $\nu$-almost all $z \in \Sigma$,

$$
\liminf _{n \rightarrow+\infty}\left|S_{n}(z)\right|=0 \text {. }
$$


When $\Gamma$ is discrete, which is our case, the above is equivalent to saying that $S_{n}(z)=0$ infinitely often in $n$.

A notable sufficient condition for cocycle recurrence was given by Schmidt:

Theorem 5.3 [Sch] Assume that $(\Sigma, F, \nu)$ is ergodic, and denote by $p_{n}$ the distribution of $S_{n} / n^{1 / d}$, i.e., for a Borel set $A$ of $\mathbb{R}^{d}$,

$$
p_{n}(A):=\nu\left(\left\{z \in \Sigma \mid \frac{S_{n}(z)}{n^{1 / d}} \in A\right\}\right) .
$$

If there exists a positive-density sequence $\left\{n_{k}\right\}_{k \in \mathbb{N}}$ and a constant $c>0$ such that

$$
p_{n_{k}}(B(0, \rho)) \geq c \rho^{d}
$$

for all sufficiently small balls $B(0, \rho)$ of center 0 and radius $\rho$ in $\mathbb{R}^{d}$, then the cocycle $\left\{S_{n}\right\}$ (equivalently, the function e) is recurrent.

Remark 5.4 In the case of interest to this paper, that is $d=2$, estabilishing the Central Limit Theorem for the family of variables $\left\{e \circ F^{k}\right\}$ (even with a degenerate limit) is clearly enough to apply Theorem 5.3. This is in fact how Schmidt proves Theorem 2.7 via $[\mathrm{BS}]$.

Coming back to the actual system at hand, this is what we know:

Proposition 5.5 If $(\Sigma, F, \nu)$ is the dynamical system introduced above then

(a) Every measurable invariant set of $\Sigma$ is of the form $\mathcal{N} \times B \bmod \nu$, where $B$ is a measurable set of $\mathcal{L}$. Furthermore, either $B$ or $\mathcal{L} \backslash B$ has empty interior.

(b) The system is topologically transitive.

(c) In the case of almost sure recurrence (that is, when $\Pi(\mathcal{R})=1$ or, which is the same, when $\left\{S_{n}\right\}$ is a recurrent cocycle), $(\Sigma, F, \nu)$ is ergodic.

Proof. For a given $\ell \in \mathcal{L}$, consider the dynamical system $\left(\mathcal{M}_{\ell}, T_{\ell}, \mu\right)$, corresponding to the $\mathrm{LG} \ell$. In view of Theorem 2.3, we construct "local stable and unstable manifolds" for $F$ at a.e. point of $\mathcal{N} \times\{\ell\}$. (More precisely, the "LUMs" are constructed as push-forwards of the LSMs of $T_{\ell}$ onto the cross-section $\mathcal{N}$; analogously, the "LUMs" are pull-backwards of the LUMs of $T_{\ell}$.) These curves are contained in $\mathcal{N} \times\{\ell\}$. The quotation marks are in order here as they are not bona fide LSUMs for $(\Sigma, F, \nu)$, which is not a hyperbolic system in any reasonable sense.

We now exploit Theorem 2.4 to conclude that, in each connected component of $\mathcal{N} \times\{\ell\}$, a.e. pair of points (w.r.t. $\mu_{1}$ ) are joined through a polyline of "LSUMs", therefore, via the usual Hopf argument, they lie in the same ergodic component of $(\Sigma, F, \nu)$. On the other hand, it is easy to verify that no two connected components 
of $\mathcal{N} \times\{\ell\}$ (each corresponding to a different segment of $\partial_{*} C_{0}$ ) can have separate dynamics, i.e., belong to distinct ergodic components. In conclusion, at least for a.a. $\ell \in \mathcal{L}, \mathcal{N} \times\{\ell\}$ is cointained in the same ergodic component of $(\Sigma, F, \nu)$. Which is to say, the only $F$-invariant sets of $\Sigma$, modulo $\nu$, are of the form $\mathcal{N} \times B$. That $B$ is measurable $\bmod \Pi$ in $\mathcal{L}$ is a consequence of Lemma A.1 of the Appendix. This proves the first part of statement (a).

Next, consider two open sets $U_{1}, U_{2} \in \Sigma$. For the purpose of proving topological transitivity one can always pass to subsets, so assume that, for $i \in\{1,2\}, U_{i}=$ $V_{i} \times B_{i}$, where $V_{i}$ is an open set of $\mathcal{N}$ and $B_{i}$ is a cylinder of $\mathcal{L}$. This means that, for each $i$, there exists a finite subset of $\Gamma$, called $\Lambda_{i}$, and a family of open sets of $\Omega$, $\left\{A_{i}^{\eta}\right\}_{\eta \in \Lambda_{i}}$, such that $B_{i}=\left\{\ell \in \mathcal{L} \mid \forall \eta \in \Lambda_{i}, \ell_{\eta} \in A_{i}^{\eta}\right\}$.

Take a sufficiently large $\gamma_{0} \in \Gamma$ so that $\left(\Lambda_{2}+\gamma_{0}\right)$ does not intersect $\Lambda_{1}$. It is clearly possible to find a periodic $\bar{\ell}=\left\{\bar{\ell}_{\gamma}\right\}$ such that, for all $\eta \in \Lambda_{1}, \bar{\ell}_{\eta} \in A_{1}^{\eta}$ and, for all $\eta \in \Lambda_{2}, \bar{\ell}_{\eta+\gamma_{0}} \in A_{2}^{\eta}$. By construction, $\bar{\ell} \in B_{1}$ and $\sigma_{\gamma_{0}}(\bar{\ell}) \in B_{2}$. By Theorem $2.7, \bar{\ell}$ is recurrent so $\left(\mathcal{M}_{\bar{\ell}}, T_{\bar{\ell}}, \mu\right)$ is ergodic. This implies that almost every billiard trajectory, in the three-dimensional phase space of $\ell$, intersects the cross-section defined by $\left\{\left(q+\gamma_{0}, v\right) \mid(q, v) \in V_{2}\right\}$. In other words, since $\mu_{1}\left(V_{1}\right)>0$, there exist a non-singular $\bar{x} \in V_{1}$ and an integer $n$ such that $F^{n}(\bar{x}, \bar{\ell}) \in V_{2} \times\left\{\sigma_{\gamma_{0}}(\bar{\ell})\right\} \subset V_{2} \times B_{2}$. But since $\bar{x}$ is non-singular and the metric on $\Omega$ is compatible with the dynamics (Definition 3.1), we can perturb $\bar{x}$ and $\bar{\ell}$ a little bit and still end up in $V_{2} \times B_{2}$. This means that there exists an open neighborhood $\mathcal{U}$ of $(\bar{x}, \bar{\ell})$ such that $F^{n}(\mathcal{U}) \subset V_{2} \times B_{2}$. This fact proves $(b)$ and the second part of $(a)$.

For (c) we use the following lemma, whose proof will be given below.

Lemma 5.6 For $\ell \in \mathcal{L}$ and $\gamma \in \Gamma$, set

$$
D_{\ell}^{\gamma}:=\left\{x \in \mathcal{N} \mid S_{n}(x, \ell)=\gamma, \text { for some } n \in \mathbb{N}\right\}
$$

and

$$
E:=\left\{\ell \in \mathcal{L} \mid \forall \gamma \in \Gamma, \mu_{1}\left(D_{\ell}^{\gamma}\right)>0\right\} .
$$

If $\Pi(E)>0$ then $(\Sigma, F, \nu)$ is ergodic.

In the hypothesis of $(c)$, a.e. $\ell$ is an ergodic Lorentz gas so, by the argument used earlier, $\mu_{1}\left(D_{\ell}^{\gamma}\right)=1$ for all $\gamma$. Thus $\Pi(E)=1$.

Q.E.D.

Proof of Lemma 5.6. Suppose the system is not ergodic. By Proposition 5.5(a), we have an invariant set $\mathcal{N} \times B(\bmod \nu)$, with $B$ a Borel set of $\mathcal{L}$ and $\Pi(B) \in(0,1)$. Set $B^{c}:=\mathcal{L} \backslash B$. Either $B$ or $B^{c}$ (or both) must intersect $E$ in a positive-measure subset. Say that this happens for $B$. Since $\left(\mathcal{L},\left\{\sigma_{\eta}\right\}, \Pi\right)$ is ergodic, one can find $O \subseteq B \cap E$ and $\gamma \in \Gamma$ such that $\Pi(O)>0$ and

$$
\sigma_{\gamma}(O) \subseteq B^{c}
$$


Fix $\ell \in O$. The hypotheses of the lemma imply that there is a positive integer $n$ and a set $D_{\ell}^{\gamma, n} \subset D_{\ell}^{\gamma}$, with $\mu_{1}\left(D_{\ell}^{\gamma, n}\right)>0$, such that $S_{n}(x, \ell)=\gamma$ for all $x \in D_{\ell}^{\gamma, n}$. That is to say,

$$
F^{n}\left(D_{\ell}^{\gamma, n} \times\{\ell\}\right) \subseteq \mathcal{N} \times \sigma_{\gamma}(\ell) \subseteq \mathcal{N} \times B,
$$

the last inclusion holding at least for a.e. $\ell \in O$, due the $F$-invariance of $\mathcal{N} \times B \bmod$ $\nu$ (notice that we have implicitly used Fubini's Theorem and Proposition 5.5(a)). This gives that $\sigma_{\gamma}(O) \subseteq B \bmod \Pi$, in contradiction with (5.4).

Q.E.D.

\section{Toy models}

As we have seen, exploring the statistical properties of $(\Sigma, F, \nu)$ is not exactly a trivial task. In this section we consider much simplified versions of that dynamical system that nonetheless have the same lattice structure. One can call this structure 'deterministic dynamics in a random enviroment'. The intent is to get an idea of those properties of the system that depend more on the random environment than on the details of the dynamics. (A less easy model will be treated in [L3].)

In these examples $\Gamma$ will always be $\mathbb{Z}^{2}$. Let us denote

$$
\mathbf{E}=g_{1}=(1,0), \quad \mathbf{N}=g_{2}=(0,1), \quad \mathbf{W}=g_{3}=(-1,0), \quad \mathbf{S}=g_{4}=(0,-1),
$$

the symbols standing for East, North, West and South. These are the primitive directions of $\mathbb{Z}^{2}$ and together they form the set $G$. To each of these directions is associated a copy of the unit square $[0,1]^{2}$. These four copies are named $\mathcal{N}_{\mathbf{E}}=\mathcal{N}_{1}$, $\mathcal{N}_{\mathbf{N}}=\mathcal{N}_{2}, \mathcal{N}_{\mathbf{W}}=\mathcal{N}_{3}$, and $\mathcal{N}_{\mathbf{N}}=\mathcal{N}_{4} ;$ also $\mathcal{N}:=\bigsqcup_{i=1}^{4} \mathcal{N}_{i}$. A point $x \in \mathcal{N}_{\mathbf{E}}$ corresponds to the particle entering $C_{0}$ from the western side (its incoming direction being $\mathbf{E}$ ), and so on analogously. We endow $\mathcal{N}$ with $\mu_{1}$, the Lebesgue measure divided by 4 , which is the right normalization factor here.

To complete the definition of our toy version of $(\Sigma, F, \nu)$, following the paradigm of Section 5, we need to introduce the probability space $(\Omega, \pi)$ that governs the randomness of each cell; the map $R_{\omega}: \mathcal{N} \longrightarrow \mathcal{N}$ that gives the dynamics in a cell in the state $\omega \in \Omega$; and the exit function $e: \mathcal{N} \times \Omega \longrightarrow G$. All these objects will vary from example to example.

Before discussing the models one by one, let us introduce the $m$-baker's map $K_{m}:[0,1]^{2} \longrightarrow[0,1]^{2}$. For $m$ a positive integer and $y:=\left(y_{1}, y_{2}\right) \in[0,1]^{2}$,

$$
K_{m}\left(y_{1}, y_{2}\right)=\left(\left\{m y_{1}\right\}, \frac{y_{2}+\left[m y_{1}\right]}{m}\right)
$$

where $[\rho]$ and $\{\rho\}$ are the integer and fractional part, respectively, of $\rho \geq 0$. Of course, $K_{2}$ is the standard baker's map. 


\subsection{Example 1: Mimicking the standard random walk}

Here $R_{\omega}$ acts on each $\mathcal{N}_{i}$ as a 4-baker's map. Precisely, if $x:=(y, i) \in \mathcal{N}$ (with $y \in[0,1]^{2}$ and $\left.i \in\{1, \ldots, 4\}\right)$, then

$$
R_{\omega}(y, i):=\left(K_{4}(y), e((y, i), \omega)\right) .
$$

Thus the dynamics does not really depend on the random state of the cell: $R$ depends on $\omega$ only through $e$, that is, only insofar as $R_{\omega}(x)$ must necessarily land on $\mathcal{N}_{e(x, \omega)}$.

In this example, $\Omega:=\{1,2,3\}$ and $\pi$ is any probability measure there (there is nothing special about the number 3 , and that is exactly the point in choosing it). The exit function $e$ is given in terms of level sets by Fig. 4. Observe that $e$ depends non-trivially on $\omega$, otherwise the system would be of a much simpler nature and recurrence would just amount to the function recurrence of $e$ relative to $\left(\mathcal{N}, R, \mu_{1}\right)$.

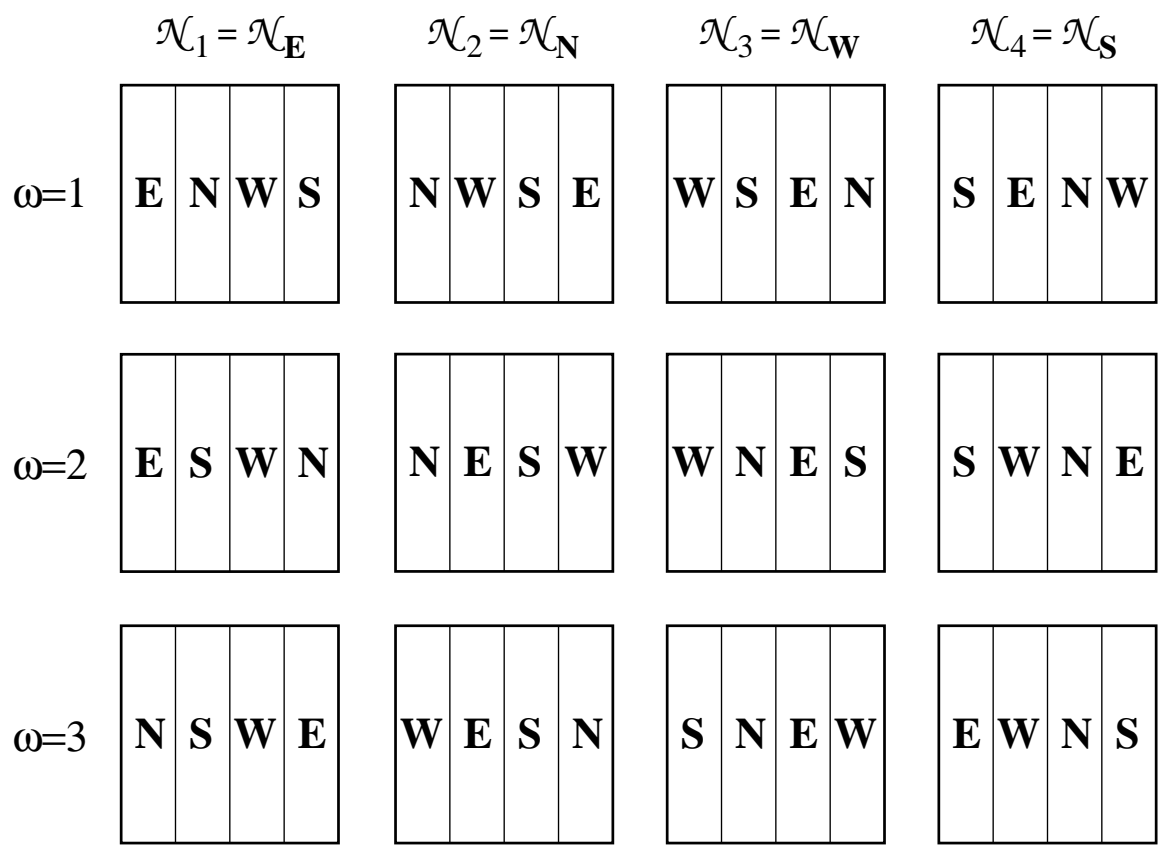

Figure 4: The definition of $e$ for Example 1. Each row displays a copy of $\mathcal{N}:=\bigsqcup_{i=1}^{4} \mathcal{N}_{i}$, corresponding to different values of $\omega \in\{1,2,3\}$. The level sets are all rectangles of base $1 / 4$ and height 1 . The label in each level set is the common image of the set via $e$, according to the notation (6.1).

Remark 6.1 Notice that, for all $\omega \in \Omega$ and $i=1, \ldots, 4, R_{\omega}$ is invertible and

$$
\mu_{1}\left(\left\{x \in \mathcal{N} \mid e(x, \omega)=g_{i}\right\}\right)=\frac{1}{4} .
$$


These facts are essential if we want to think of our dynamical system as generated by a "physical" (read: conservative and invertible) system $(\mathcal{M}, T, \mu)$, as described in Section 5. The same will occur also for Examples 2 and 3.

We claim that in this setup the particle moves as in a standard random walk for every realization of the random enviroment. In more exact terms, for every $\ell \in\left\{1,2,3 \mathbb{Z}^{2}\right.$, the stochastic process

$$
\left(\mathcal{N}, \mu_{1}\right) \ni x \longmapsto\left\{S_{n}(x, \ell)\right\}_{n \geq 0}=\left\{\sum_{k=0}^{n-1}\left(e \circ F^{k}\right)(x, \ell)\right\}_{n \geq 0}
$$

is a standard random walk. In fact, if $\left\{\gamma_{k}\right\}_{k=0}^{n}$ is a path in $\mathbb{Z}^{2}$ (i.e., $\gamma_{0}=0$ and $\left.\left|\gamma_{k+1}-\gamma_{k}\right|=1 \forall k\right)$ and $i \in\{1, \ldots, 4\}$, one realizes that the conditional probability

$$
\mu_{1}\left(\left(e \circ F^{n}\right)(\cdot, \ell)=g_{i} \mid S_{k}(\cdot, \ell)=\gamma_{k}, \forall k=1, \ldots, n\right)=\frac{1}{4} .
$$

(The set in which we condition is a rectangle of base $4^{-n+1}$ and height 1 . The preimages of $e \circ F^{n}$ will subdivide it into 4 rectangles of base $4^{-n}$ and height 1 , one for each $g_{i}$.)

Recurrence is thus guaranteed in every fiber $\mathcal{N} \times\{\ell\}$, which is an even stronger statement than we sought.

\subsection{Example 2: Left-Right random walk}

The previous example was indeed much too easy, and we did not utilize at all the considerations of Section 5. Example 2 is going to be a tad more involved. Here $\Omega=\{1,2\}$ and, once again, the choice of $\pi$ is irrelevant; $e$ is given by Fig. $5 . R_{\omega}$ acts as the standard baker's map, and its dependence on $\omega$ is as trivial as in the previous model:

$$
R_{\omega}(y, i):=\left(K_{2}(y), e((y, i), \omega)\right) .
$$

Reasoning along the same lines as in Section 6.1, we see that, fixing $\ell \in \Omega^{\mathbb{Z}^{2}}$ and letting $x$ range randomly in $\mathcal{N}$ according to $\mu_{1}$, we obtain the so-called LeftRight random walk. This is the stochastic process in a which a particle moves in $\mathbb{Z}^{2}$ turning its direction by 90 degrees after every step, with a fifty-fifty chance of turning left or right (left and right being relative to the direction of the motion; the absolute directions in $\mathbb{Z}^{2}$ are $\mathbf{W}, \mathbf{E}$ and so on).

What makes this model more complicated than Example 1 is that it is not a Markov chain (at least not in its simplest formulation, that is, as a random process whose $n^{\text {th }}$ component is the position of the particle at time $n$ ). Not that probabilists have a hard time proving the recurrence of this model, but here we will do so by applying Theorem 5.3. 


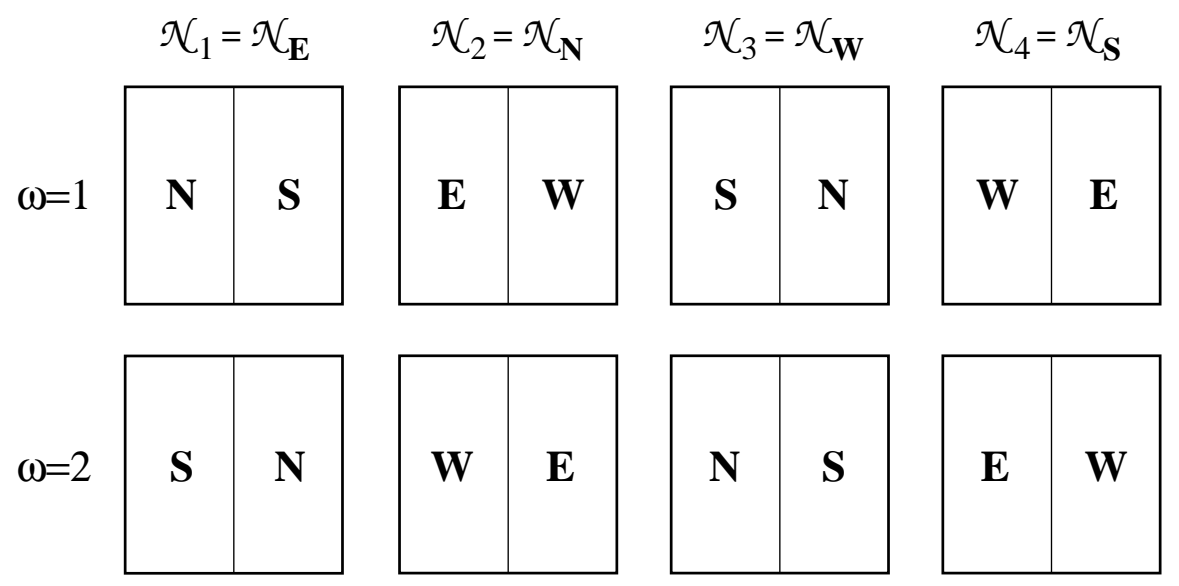

Figure 5: The definition of $e$ for Example 2. See caption of Fig. 4 for explanations.

First of all, we claim that $(\Sigma, F, \nu)$ is ergodic. This might not be so apparent, as the dynamics has an obvious symmetry. In fact, denote $\mathcal{N}^{(1)}:=\mathcal{N}_{\mathbf{E}} \sqcup \mathcal{N}_{\mathbf{W}}$ and $\mathcal{N}^{(2)}:=\mathcal{N}_{\mathbf{N}} \sqcup \mathcal{N}_{\mathbf{S}}$. For a typical $\ell \in \mathcal{L}$, the evolution of $\mathcal{N}^{(1)} \times\{\ell\}$ is always separated from $\mathcal{N}^{(2)} \times\{\ell\}$ because, if $x \in \mathcal{N}^{(1)}$, it is easy to check from Fig. 5 that $F^{n}(x, \ell)$ belongs to $\mathcal{N}^{(1)} \times \mathcal{L}$ or $\mathcal{N}^{(2)} \times \mathcal{L}$ depending on $n$ begin even or odd, respectively; but in order for the point to come back to the same cell (i.e., $F^{n}(x, \ell) \in \mathcal{N} \times\{\ell\}$ ) $n$ must be even, and therefore $F^{n}(x, \ell) \in \mathcal{N}^{(1)} \times\{\ell\}$.

This notwithstanding, that $(\Sigma, F, \nu)$ is ergodic can be seen as follows: For $j=$ 1,2 , define $\Sigma^{(i)}:=\mathcal{N}^{(j)} \times \mathcal{L}$ and consider the dynamical system $\left(\Sigma^{(j)}, F^{2}, \nu / 2\right)$. It is easy to ascertain that the "horizontal" fibers $\mathcal{N}^{(j)} \times\{\ell\}$ are wholly contained in the ergodic components of the system. Then we can use Lemma 5.6 with

$$
\Gamma=\mathbb{Z}_{\text {even }}^{2}:=\left\{\gamma=\left(\gamma_{1}, \gamma_{2}\right) \in \mathbb{Z}^{2} \mid \gamma_{1}+\gamma_{2} \in 2 \mathbb{Z}\right\}
$$

to conclude that $\left(\Sigma^{(j)}, F^{2}, \nu / 2\right)$ is ergodic. (The hypothesis of the lemma applies as it is clear that, for all $\ell \in \mathcal{L}$, one can reach any $\gamma \in \mathbb{Z}_{\text {even }}^{2}$ for some - thus many-initial conditions $x \in \mathcal{N}^{(j)}$. The proof works because $\left(\mathcal{L},\left\{\sigma_{\eta}\right\}_{\eta \in \Gamma}, \Pi\right)$ is ergodic for $\Gamma=\mathbb{Z}_{\text {even }}^{2}$ as well.) Now, if $U$ is an $F$-invariant subset of $\Sigma \bmod \nu$, set $U^{(j)}:=U \cap \Sigma^{(j)}$. Clearly $F U^{(1)}=U^{(2)}$ and $F^{2} U^{(1)}=U^{(1)}(\bmod \nu)$. Hence, both $U^{(j)}$ have either measure zero or full measure in $\Sigma^{(j)}$, whence the ergodicity of $(\Sigma, F, \nu)$.

Now, for every $\ell$, it is obvious that the projections of $S_{n}(\cdot, \ell)$ onto the horizontal and vertical directions of $\mathbb{Z}^{2}$ are independent one-dimensional random walks (at times $[n / 2]$ or $[n / 2]+1)$, which verify the $1 \mathrm{D}$ Central Limit Theorem for $n \rightarrow+\infty$. Their orthogonal sum must then verify the 2D Central Limit Theorem, which implies the same for the "more random" process $S_{n}(\cdot, \cdot)$. This, together with the ergodicity of $(\Sigma, F, \nu)$, gives the recurrence via Theorem 5.3, 


\subsection{Example 3: Deterministic walk in a random environ- ment}

The next and last example shows that recurrence in $\mathcal{L}$ is not due solely to the chaotic nature of the dynamics (which tends to produce a diffusive behavior in every LG), but may also be a consequence of the random environment. To demonstrate this point, which makes Conjecture 4.1 all the more convincing, we take $R$ to be as regular as it can be, practically the identity. Let us define

$$
R_{\omega}(y, i):=(y, e(i, \omega)) ;
$$

that is, $y$ remains constant and plays absolutely no role, not even on the exit function $e$, which, for a given state $\omega$ of the cell, depends only on the incoming direction $i$. For all practical purposes, then, each $\mathcal{N}_{i}$ can be collapsed to a point. We actually do so and for the remainder of the section we consider $\Sigma:=\{1,2,3,4\}$ or, equivalently, $\Sigma:=\{\mathbf{E}, \mathbf{N}, \mathbf{W}, \mathbf{S}\}$. Instead of a 'dynamics in a random environment', we have a 'walk in a random environment'.

Let $\Omega:=\{1,2,3,4\}$ and, for $\omega \in \Omega$,

$$
e(i, \omega):=i+\omega \bmod 4,
$$

where 'mod 4' means the congruent integer between 1 and 4 . If we rename the elements of $\Omega$ as $\mathbf{L}:=1, \mathbf{B}:=2, \mathbf{R}:=3$ and $\mathbf{F}:=4$ (the symbols standing for Forward, Backward, Left and Right), we see that every time the particle reaches a cell in the state $\omega$, it will take a step in the direction indicated by $\omega$ (relative to the incoming direction). Let us denote $\pi_{\mathbf{L}}, \pi_{\mathbf{B}}, \pi_{\mathbf{R}}, \pi_{\mathbf{F}}$ the probabilities of each of the four symbols.

It is clear that a walk is recurrent if and only if it is a periodic orbit of $(\Sigma, F, \nu)$. It is also clear that Theorem 4.2 does not hold - or better, its proof does not apply: recurrence here is not a translation invariant property. The same for Proposition 5.5 (a) and the analog of Proposition 2.6: the existence of a closed walk tells us nothing about the other walks. We collect what we know in a proposition.

Proposition 6.2 Using the notation of the previous sections on the system $(\Sigma, F, \nu)$ defined above,

(a) $\Pi(\mathcal{R})>0$.

(b) There exists a number $p_{c} \in(1 / 2,1)$ such that, if $\pi_{\mathbf{B}}>p_{c}$, or $\pi_{\mathbf{L}}>p_{c}$, or $\pi_{\mathbf{R}}>p_{c}$, then $\Pi(\mathcal{R})=1$.

(c) If $\pi_{\mathbf{L}}+\pi_{\mathbf{R}}=1$ (and thus $\pi_{\mathbf{B}}=\pi_{\mathbf{F}}=0$ ), then $\Pi(\mathcal{R})=1$.

Proof. Part (a) is obvious since, for every initial direction $i$, one can always fix $\ell_{\gamma}$ for a finite number of cells near the origin so as to create a periodic orbit. The LGs $\ell$ with those components fixed form a positive-measure cylinder. 
As for $(b), p_{c}$ is the critical probability for the site percolation in $\mathbb{Z}^{2}$ (believed to be approximately 0.59 ). If the probability of a given $\omega$ is bigger than this number, there is almost surely a closed loop of cells marked $\omega$ that surrounds the origin $[G]$. If $\omega \in \mathbf{B}, \mathbf{L}, \mathbf{R}$, this prevents any path starting at the origin from reaching infinity (cf. $[\mathrm{BT}]$ ).

Statement (c) is Theorem 2(ii) of [BT].

Q.E.D.

\section{A Appendix: A lemma from measure theory}

Lemma A.1 Let $(X, \mathcal{A}, \mu)$ and $(Y, \mathcal{B}, \nu)$ be two probability spaces, and let $\mathcal{A} \otimes \mathcal{B}$ denote the $\sigma$-algebra on $X \times Y$ generated by the rectangles (i.e., sets of the type $A \times B$, with $A \in \mathcal{A}, B \in \mathcal{B})$.

If $A \in \mathcal{A}$, with $\mu(A)>0$, and $A \times B \in \mathcal{A} \otimes \mathcal{B}$, then there exists a $B_{0} \in \mathcal{B}$ such that $B \Delta B_{0}$ is a subset of a $\nu$-null-measure set. In particular, if $(Y, \mathcal{B}, \nu)$ is complete, then $B \in \mathcal{B}$.

Proof of Lemma A.1. We first notice that, for every $C \in \mathcal{A} \otimes \mathcal{B}$, there exists a sequence $\left\{U_{n}\right\}$, where $U_{n}$ is a finite union of rectangles, such that $\lim _{n \rightarrow+\infty}(\mu \times$

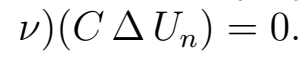

We prove the above by showing that the class $\mathcal{C}$ of such sets $C$ that can be approximated by finite unions of rectangles is a $\sigma$-algebra in $\mathcal{A} \otimes \mathcal{B}$. Since $\mathcal{C}$ contains the rectangles, it must be the whole $\mathcal{A} \otimes \mathcal{B}$.

Obviously $\mathcal{C}$ is closed by complementation (an approximating sequence for $C^{c}$ being $U_{n}^{c}$, also a finite unione of rectangles) and by countable disjoint union (it suffices to neglect the sets in the "tail" of the countable union).

So, let $U_{n}$ be an approximating sequence for $A \times B$. For $y \in Y$, denote by

$$
S_{y, n}:=\left\{x \in A \mid(x, y) \in U_{n}\right\}
$$

the section of $U_{n} \cap(A \times Y)$ relative to $y$. Set $m_{n}(y):=\mu\left(S_{y, n}\right)$. This is clearly a measurable function $Y \longrightarrow \mathbb{R}_{0}^{+}$(it is actually a simple function). Since it is also bounded, then $m_{n} \in L^{1}(Y, \nu)$.

Assume for the moment that $x \in A, y \in B$. Then $(x, y) \in(A \times B) \Delta U_{n} \Longleftrightarrow$ $(x, y) \notin U_{n} \Longleftrightarrow x \notin S_{y, n}$. Now assume that $x \in A$, but $y \notin B$. By the same token $(x, y) \in(A \times B) \Delta U_{n} \Longleftrightarrow(x, y) \in U_{n} \Longleftrightarrow x \in S_{y, n}$. These considerations, combined with Fubini's Theorem, give

$$
\begin{aligned}
& (\mu \times \nu)\left[\left((A \times B) \Delta U_{n}\right) \cap(A \times Y)\right]= \\
= & \int_{B} \mu\left(A \backslash S_{y, n}\right) d \nu(y)+\int_{B^{c}} \mu\left(S_{y, n}\right) d \nu(y)= \\
= & \int_{Y}\left|m_{n}(y)-\mu(A) \chi_{B}(y)\right| d \nu(y) .
\end{aligned}
$$


Therefore $m_{n} \rightarrow \mu(A) \chi_{B}$ in $L^{1}(Y, \nu)$, as $n \rightarrow+\infty$. For a subsquence, then-let us call it again $\left\{m_{n}\right\}$ - the convergence occurs almost everywhere. More precisely, there exists a set $Y_{0}$, with $\nu\left(Y_{0}\right)=1$, such that, for every $y \in Y_{0}$,

$$
\lim _{n \rightarrow+\infty} m_{n}(y)=\mu(A) \chi_{B}(y) .
$$

For a fixed $\rho \in(0, \mu(A))$, we denote by $L_{n}:=\left\{y \in Y \mid m_{n}(y) \geq \rho\right\}$ a certain filled level set of $m_{n}$. Then (A.3) implies that

$$
Y_{0} \cap \bigcup_{m \in \mathbb{N}} \bigcap_{n \geq m} L_{n}=Y_{0} \cap B .
$$

Setting $B_{0}:=Y_{0} \cap B \in \mathcal{B}$ concludes the proof of the lemma.

Q.E.D.

\section{References}

[A] J. Aaronson, An introduction to infinite ergodic theory, Americal Mathematical Society, Providence, RI, 1997.

[BCST] P. BÁlint, N. Chernov, D. SzÁsz and I. P. Tóth, Multi-dimensional semi-dispersing billiards: singularities and the fundamental theorem, Ann. Henri Poincaré 3 (2002), no. 3, 451-482.

[BS] L. A. Bunimovich And YA. G. Sinai, Statistical properties of Lorentz gas with periodic configuration of scatterers, Comm. Math. Phys. 78 (1980/81), no. 4, 479-497.

[BT] L. A. Bunimovich And S. E. Troubetzkoy, Recurrence properties of Lorentz lattice gas cellular automata, J. Statist. Phys. 67 (1992), no. 1-2, 289-302.

[CM] N. I. Chernov AND R. Markarian, Introduction to the ergodic theory of chaotic billiards, 2nd ed., Publicações Matemáticas do IMPA, Instituto de Matemática Pura e Aplicada, Rio de Janeiro, 2003.

[CY] N. I. Chernov And L.-S. Young, Decay of correlations for Lorentz gases and hard balls, in: Hard ball systems and the Lorentz gas, D. Szász (ed.), EMS 101, SpringerVerlag, Berlin-New York, 2000.

[Co] J.-P. Conze, Sur un critère de récurrence en dimension 2 pour les marches stationnaires, applications, Ergodic Theory Dynam. Systems 19 (1999), no. 5, 1233-1245.

[G] G. Grimmett, Percolation, 2nd ed., Springer-Verlag, Berlin, 1999.

[KS] A. Katok and J.-M. Strelcyn (in Collaboration with F. Ledrappier and F. PRZYTYCKI), Invariant manifolds, entropy and billiards; smooth maps with singularities, LNM 1222, Springer-Verlag, Berlin-New York, 1986.

[L1] M. LencI, Semi-dispersing billiards with an infinite cusp I, Comm. Math. Phys. 230 (2002), no. 1, 133-180.

[L2] M. LencI, Aperiodic Lorentz gas: recurrence and ergodicity, Ergodic Theory Dynam. Systems 23 (2003), no. 3, 869-883.

[L3] M. LENCI, A random walk in a random environment mimicking a Lorentz gas, In preparation. 
[Sch] K. Schmidt, On joint recurrence, Comptes Rendu Acad. Sci. Paris Sér. I Math. 327 (1998), no. 9, 837-842.

[SC] YA. G. SinAI AND N. I. Chennov, Ergodic properties of certain systems of twodimensional discs and three-dimensional balls, Russ. Math. Surveys 42 (1987), no. 3, 181-207. 